Rapid Communications

\title{
Determination of 3-Methyl-quinoxaline-2-carboxylic Acid and Quinoxaline-2- carboxylic Acid in Pork Based on a Background Fluorescence Quenching Immunochromatographic Assay
}

\author{
Xulin WAN, Xin WANG, and Xiaoqi TAO ${ }^{\dagger}$ \\ College of Food Science, Southwest University, Chongqing, 400715, China
}

\begin{abstract}
A novel rapid method based on a background fluorescence quenching immunochromatographic assay (bFQICA) was established to achieve simultaneously the quantitative detection of 3-methyl-quinoxaline-2-carboxylic acid (MQCA) and quinoxaline-2-carboxylic acid (QCA), which were efficiently extracted and enriched 4 times using immunomagnetic beads from pork. The analysis of field pork samples by bFQICA was in accordance with that of LC-MS/MS; especially, the proposed bFQICA exhibited great advantages in convenience and efficiency, which only takes 30 min for the detection of MQCA and QCA.
\end{abstract}

Keywords Background fluorescence quenching immunochromatographic assay (bFQICA), 3-methyl-quinoxaline-2carboxylic acid (MQCA), quinoxaline-2-carboxylic acid (QCA), immunomagnetic beads, pork

(Received May 26, 2020; Accepted June 12, 2020; Advance Publication Released Online by J-STAGE June 19, 2020)

Olaquindox (OLA) and carbadox (CBX) (Fig. S1, Supporting Information) are the well-known members of the quinoxaline antibacterial, ${ }^{1}$ which have been widely used as medicinal feed additives and growth promoters for the prevention dysentery and bacterial enteritis in swine. ${ }^{2}$ However, OLA and CBX can be rapidly metabolized and converted into a marker of drug residue in animal tissues, 3-methyl-quinoxaline-2-carboxylic acid (MQCA) and quinoxaline-2-carboxylic acid (QCA), respectively., ${ }^{2,3}$ Moreover, MQCA and QCA have carcinogenic, mutagenic and photo allergenic effects on people, threating the food safety. ${ }^{4}$

Indeed, many strategies have been devoted to establish sensitive, fast and reliable analytical methods applicable to determine OLA, CBX and their metabolites (MQCA and QCA), such as high-performance liquid chromatography ${ }^{2}$ and highperformance liquid chromatography-tandem mass spectrometry (HPLC-MS/MS). ${ }^{5}$ However, it has limitations in concerning food testing because of expensive instruments, a long sample preparation time and professional knowledge of operators. In addition, immunoassay is considered as to be a rapid technique for veterinary drug residue analysis with high throughput tests. ${ }^{6,7}$ Some strategies were proposed, such as time-resolved fluoroimmunoassay (TR-FIA) ${ }^{8}$ dual-label direct competitive fluorescence-linked immunosorbent assay (dc-FLISA) ${ }^{9}$ and enzyme-linked immunosorbent assay (ELISA) ${ }^{10}$ but these methods required tedious washing steps and long times for consumption. The traditional colloidal gold immunochromatographic assay (GICA) $)^{11,12}$ is fast, simple and low cost, but always shows disadvantages of a narrow quantitative range or just a qualified determination. In this study, we firstly proposed a quantitative analysis method for a background fluorescence quenching immunochromatography assay (bFQICA) ${ }^{13}$ with immunomagnetic beads separation and enrichment based on a streptavidin-biotin system for the simultaneous determination of MQCA and QCA residues in pork (Fig. 1). This method utilizes the ability of

$\doteqdot$ To whom correspondence should be addressed.

E-mail:77179000@qq.com gold nanoparticles to efficiently quench fluorescence, and to measure the background fluorescence value by the instrument to achieve the rapid quantitative detection of analytes. ${ }^{14}$ In addition, immunomagnetic beads capturing could decrease the sample pretreatment time, increase the assay sensitivity and address the any interferences from complex samples. ${ }^{15,16}$

A schematic illustration of the bFQICA strip is shown in Fig. 2. AuNPs-anti-MQCA mAb immunoprobes were bound to MQCA and/or QCA in the standard, or samples extraction solution; the mixture was dripped onto the sample pad and could move towards the absorbent pad through a capillarity. Based on the competitive binding format, when there were no MQCA and QCA (negative) in the solution, AuNPs-anti-MQCA $\mathrm{mAb}$ immunoprobes bound to the MQCA- $\mathrm{NH}_{2}-\mathrm{OVA}$ immobilized on the NC membrane and the gathered AuNPs could obviously quench the fluorescence of $\mathrm{T}$ line (F2). The remaining AuNPs-anti-MQCA mAb immunoprobes continued to move towards to the $\mathrm{C}$ line and combined with the goat antimouse $\mathrm{IgG}$, resulting in less fluorescence quenching at the $\mathrm{C}$ line (F1). At this point, the ratio of F1/F2 was minimum (min). Conversely, when MQCA and/or QCA (positive) were present, the AuNPs-anti-MQCA mAb immunoprobes bound with the analytes, resulting in a decreased fluorescence quenching degree of the T line. Moreover, these probes (the unbound AuNPs-antiMQCA mAb immunoprobes and AuNPs-anti-MQCA mAbanalytes complex) could combine with goat anti-mouse IgG at $\mathrm{C}$ line and obviously quenched the fluorescence of $\mathrm{C}$ line. At this point, the ratio of F1/F2 was maximum (max). The value of the fluorescence signal ratio $(\mathrm{F} 1 / \mathrm{F} 2)$ decreased with the increased concentration of analytes present in the standards or sample solution. Interestingly, F1 waxed and F2 waned with increased concentration of the analytes.

Under the optimized conditions (Supporting Information), the standard solutions of MQCA were diluted in $0.02 \mathrm{M}$ PBS to produce a concentration range from 0 to $6.4 \mu \mathrm{g} \mathrm{L}^{-1}(0,0.1,0.2$, $0.4,0.8,1.6,3.2,6.4 \mu \mathrm{g} \mathrm{L}^{-1}$ ), and were measured by the developed bFQICA strip. Two arrows indicate the position of the $\mathrm{T}$ line 


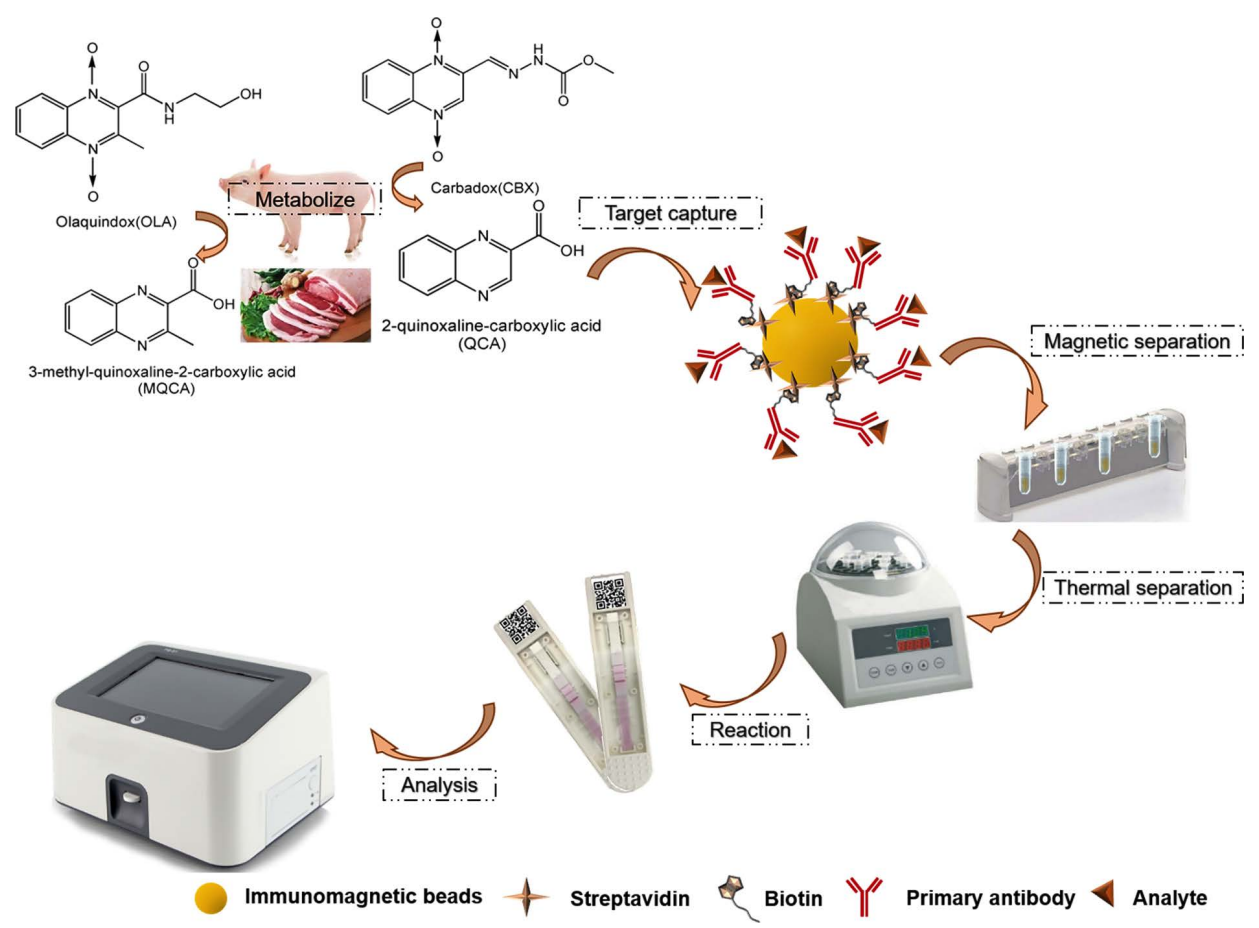

Fig. 1 Schematic demonstration of MQCA and QCA detection procedures by bFQICA.

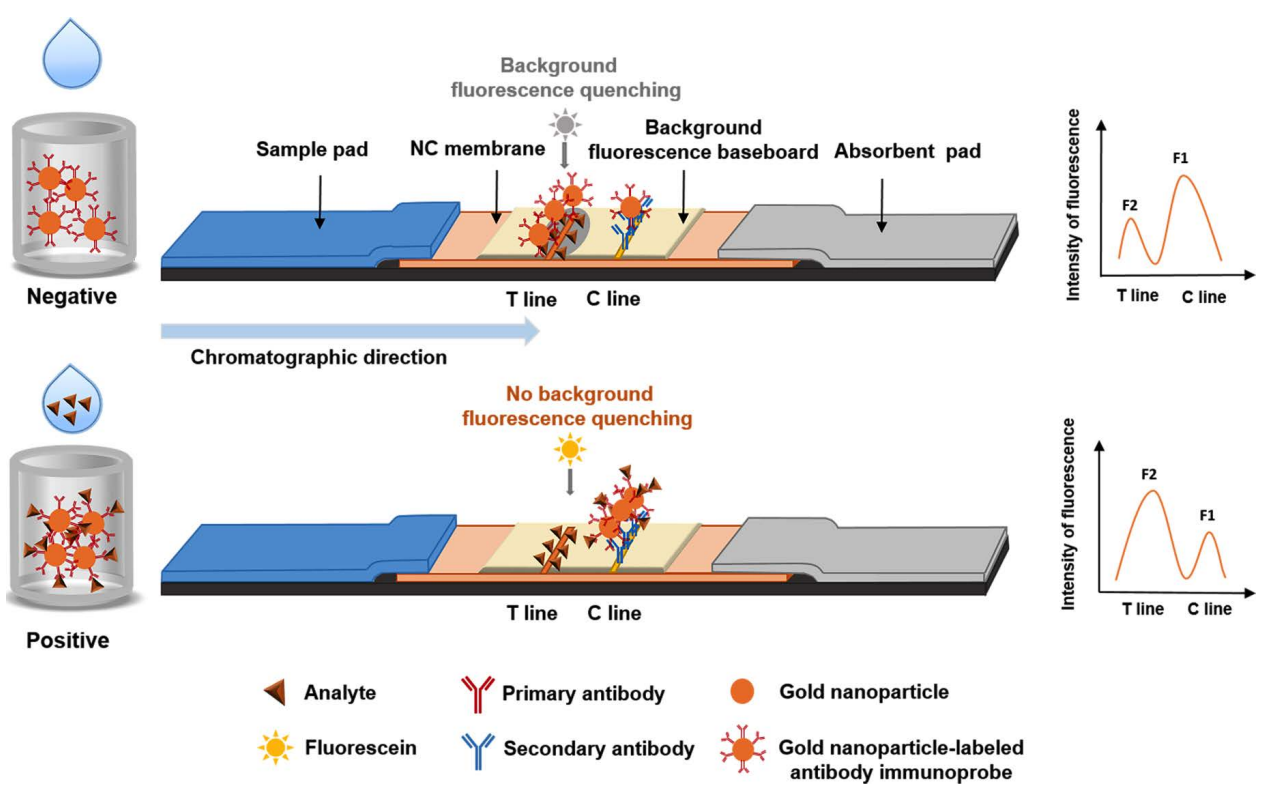

Fig. 2 Schematic representation of bFQICA for the quantitative determination of MQCA and QCA.

and $\mathrm{C}$ line on strips, and could serve as a quick, qualitative determination by eyes according to the color intensity (Fig. 3a). The color intensity gradient from low to high represented increasing aggregation of AuNPs-anti-MQCA mAb probes, and a decreasing value of the fluorescence signal. The regression equation was $y=1.518+(6.706-1.518) /\left(1+(x / 0.356)^{2.273}\right)$, with a correlation coefficient of 0.9636 (Fig. 3b). The sensitivity of bFQICA was evaluated by using the $\mathrm{IC}_{50}$ value of the obtained calibration curve, which was $0.34 \mu \mathrm{g} \mathrm{L}^{-1}$, and the dynamic linear range determined as the concentrations causing $20-80 \%$ inhibition of $\mathrm{F} 1 / \mathrm{F} 2$ was $0.1-1.6 \mu \mathrm{g} \mathrm{L}^{-1}$. This performance was superior to that of reported studies in terms of the sensitivity. ${ }^{17,18}$ Matrix elimination was considered in the proposed bFQICA method for MQCA/QCA detection. MQCA and QCA in pork samples were extracted and enriched by immunomagnetic beads (Fig. 1) ${ }^{19}$ and separated from the sample interference matrix by a magnetic field, and then the analytes (MQCA/QCA) were eluted into $0.02 \mathrm{M} \mathrm{PB}$ by heating at $85^{\circ} \mathrm{C}$. The superimposition of the calibration curves indicated that there was no significant matrix effect (Fig. S2). Therefore, the pretreatment of immunomagnetic beads can basically eliminate matrix interference, and the pork sample can be analyzed using the standard inhibition curve instead of the sample matrix curve. The LOD was calculated as the mean value of 20 blank pork samples plus three-times the standard deviation (mean + 3SD). The LODs of the developed bFQICA were $0.03 \mu \mathrm{g} \mathrm{kg}^{-1}$ for MQCA and 
$\begin{array}{lllllll}\text { (a) } \mathrm{MQCA} / \mu \mathrm{\mu g} \mathrm{L}^{-1} & 0 & 0.2 & 0.4 & 0.8 & 1.6 & 6.4\end{array}$

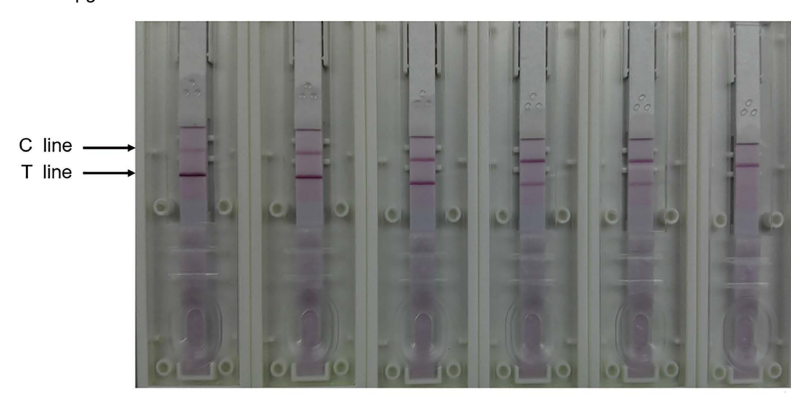

(b)

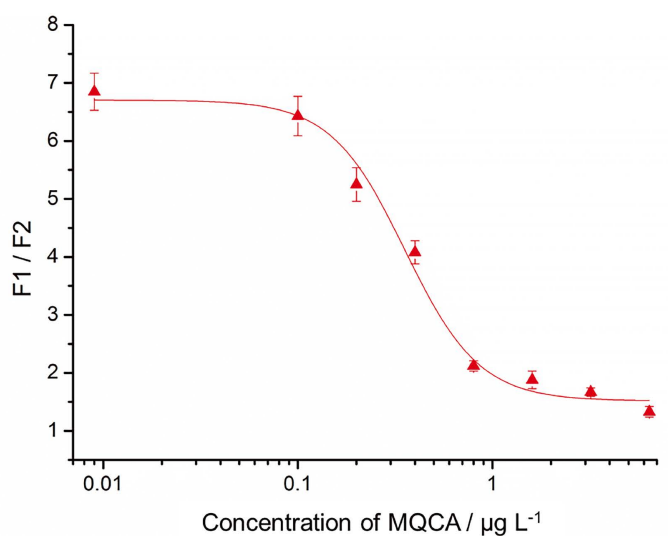

Fig. 3 (a) Typical responses of the bFQICA strips to MQCA with different concentrations; (b) standard curves of the bFQICA for MQCA

$0.075 \mu \mathrm{g} \mathrm{kg}^{-1}$ for QCA in pork. Compared with the previously reported immunoassay, the bFQICA not only possessed a lower LOD, but also took less detection time (Table S1). To evaluate the accuracy of the developed bFQICA, blank samples confirmed by $\mathrm{LC}-\mathrm{MS} / \mathrm{MS}^{20}$ were fortified at $0.03,0.06$ and $0.12 \mu \mathrm{g} \mathrm{kg}^{-1}$ with MQCA, and $0.075,0.15$ and $0.30 \mu \mathrm{g} \mathrm{kg}^{-1}$ with QCA prior to analysis, respectively. The average intra-assay and interassay recoveries of MQCA and QCA at the spiked levels in pork ranged from 92.4 to $107.0 \%$, with coefficients of variation of $<15 \%$ (Table S2). These confirmed that the bFQICA was suitable for the detection of MQCA and QCA residues in pork.

To evaluate the reliability of the developed bFQICA method, 20 field pork samples were analyzed with the developed bFQICA and LC-MS/MS. ${ }^{20}$ As shown in Table S3, the results of field pork samples from these two methods were coincident (Sample P2 and negative samples). In addition, the result measured by bFQICA may underestimate the MQCA+QCA residue concentrations when compared to those produced by the LC-MS/MS (Sample P17). This underestimation occurred because the anti-MQCA mAb has a CR of $40.0 \%$ for QCA (Table S4). The limitation of bFQICA did not affect its usefulness as a screening tool because it still detected the presence of MQCA+QCA above their detection limits, suggesting that the established bFQICA could screen MQCA and QCA in the incurred samples as well as LC-MS/MS.

In summary, bFQICA for the quantitative determination of MQCA and QCA in pork was successfully developed. This method combines both advantages of GICA and FLISA, which are fast, sensitive and quantifiable. MQCA and QCA can be specifically extracted and enriched for 4 times by immunomagnetic beads from pork, and the overall detection time was greatly reduced to only $30 \mathrm{~min}$. Interestingly, F1 (the fluorescence of $\mathrm{C}$ line) waxed and $\mathrm{F} 2$ (the fluorescence of $\mathrm{T}$ line) waned with increased concentration of the analytes, increasing the sensitivity. In addition, the concentration of analytes can be directly displayed by the built-in QR-code through instrument recognition, which is efficient and convenient. Thus, this newly developed bFQICA strategy is a promising approach for the rapid field detection of MQCA and QCA in animal-derived foods.

\section{Acknowledgements}

This research was funded by the National Natural Science Foundation of China, grant number 31672605; Natural Science Foundation of Chongqing, China, grant number cstc2018jcyjAX0242 and cstc2017jcyjAX0313; China Postdoctoral Science Foundation, grant number 2016M590855; Chongqing Postdoctoral Science Foundation Special Funded Project, grant number Xm2017074.

\section{Supporting Information}

It contains detailed experimental procedures in this study and supplementary figures and tables. This material is available free of charge on the Web at http://www.jsac.or.jp/analsci/.

\section{References}

1. Z. Y. Liu and Z. L. Sun, Med. Chem., 2013, 9, 1017.

2. Z. Duan, J. Yi, G. Fang, L. Fan, and S. Wang, Food Chem., 2013, 139, 274.

3. B. Yang, L. Huang, Y. Wang, Y. Liu, Y. Tao, D. Chen, Z. Liu, K. Fang, Y. Chen, and Z. Yuan, J. Agric. Food Chem., 2010, 58, 937.

4. K. Zhang, M. Ban, Z. Zhao, H. Zheng, X. Wang, M. Wang, C. Fei, and F. Xue, Res. Vet. Sci., 2012, 93, 1393.

5. J. O. Boison, S. C. Lee, and R. G. Gedir, Anal. Chim. Acta, 2009, 637, 128.

6. C. Chafer-Pericas, A. Maquieira, and R. Puchades, TracTrends Anal. Chem., 2010, 29, 1038.

7. M. Meng and R. M. Xi, Anal. Lett., 2011, 44, 2543.

8. T. Le, H. Yu, and X. Niu, Food Chem., 2015, 175, 85.

9. T. Le, L. Zhu, and H. Yu, Food Chem., 2016, 199, 70

10. W. Jiang, R. C. Beier, Z. Wang, Y. Wu, and J. Shen, J. Agric. Food Chem., 2013, 61, 10018.

11. T. Le, J. Xu, Y. Y. Jia, H. Q. He, X. D. Niu, and Y. Chen, Food Addit. Contam. A, 2012, 29, 925.

12. D. Peng, X. Zhang, Y. Wang, Y. Pan, Z. Liu, D. Chen, F. Sheng, and Z. Yuan, Food Chem., 2017, 237, 290.

13. X. Chen, Y. Xu, J. Yu, J. Li, X. Zhou, C. Wu, Q. Ji, Y. Ren, L. Wang, Z. Huang, H. Zhuang, L. Piao, R. Head, Y. Wang, and J. Lou, Anal. Chim. Acta, 2014, 841, 44.

14. B. B. Li, J. Z. Song, J. Chen, L. Ma, X. X. Li, J. T. Li, and M. Guan, Anal. Lett., 2019, 52, 1340.

15. F. Song, Y. Zhou, Y. S. Li, X. M. Meng, X. Y. Meng, J. Q. Liu, S. Y. Lu, H. L. Ren, P. Hu, Z. S. Liu, Y. Y. Zhang, and J. H. Zhang, Food Chem., 2014, 158, 445.

16. F. Xie, W. Lai, J. Saini, S. Shan, X. Cui, and D. Liu, Food Chem., 2014, 150, 99.

17. G. Li, L. Zhao, F. Zhou, J. Li, Y. Xing, T. Wang, X. Zhou, B. Ji, and W. Ren, Food Chem., 2016, 209, 279.

18. X. Y. Pei, Q. Wang, X. M. Li, J. Xie, S. L. Xie, T. Peng, C. Wang, Y. Z. Sun, and H. Y. Jiang, Food Anal. Methods, 2016, 9, 1919.

19. Y. Q. Zhang, S. Zhou, X. X. Chang, and X. Q. Tao, Anal. Sci., 2019, 35, 1291

20. A. Merou, G. Kaklamanos, and G. Theodoridis, $J$. Chromatogr. B, 2012, 881, 90. 